\title{
Ubiquitin-modified hepatitis B virus core antigen effectively facilitates antigen presentation and enhances cytotoxic $T$ lymphocyte activity via the cytoplasmic transduction peptide in vitro
}

\author{
LINLIN SONG, MENG ZHUO, YUYAN TANG, XIAOHUA CHEN, YONGSHENG YU, \\ ZHENGHAO TANG and GUOQING ZANG
}

\begin{abstract}
Department of Infectious Disease, Shanghai JiaoTong University Affiliated Sixth People's Hospital, Shanghai 200233, P.R. China
\end{abstract}

Received April 23, 2014; Accepted January 22, 2015

DOI: $10.3892 / \mathrm{mmr} .2015 .3352$

\begin{abstract}
Cluster of differentiation (CD) $8^{+}$cytotoxic $\mathrm{T}$ lymphocytes (CTLs) have a key role in the elimination of hepatitis B virus (HBV)-infected cells. Ubiquitin (Ub) functions as a marker for protein degradation, which may promote the generation of peptides appropriate for major histocompatibility complex class I presentation, while the HBV core antigen $(\mathrm{HBcAg})$ possesses marked immunogenic properties. However, it remains to be elucidated whether Ub-modified $\mathrm{HBcAg}$ is able to effectively elicit significant $\mathrm{CD}^{+} \mathrm{CTL}$ activity. In order to address this issue, a prokaryotic vector was constructed to express the Ub-HBcAg-cytoplasmic transduction peptide (CTP). The fusion protein was successfully expressed and subsequently pulsed into bone-marrow-derived dendritic cells (DCs). It was confirmed that with assistance from the cell-penetrating properties of CTP, the fusion protein was able to directly penetrate into the cytoplasm of DCs. The results revealed that the $\mathrm{Ub}-\mathrm{HBcAg-CTP}$ fusion protein not only increased the expression of surface molecules in DCs and cytokine secretion from proliferating $\mathrm{T}$ cells, but also induced $\mathrm{T}$ cells to differentiate into specific CTLs and enhanced their antiviral ability. In conclusion, the Ub-HBcAg-CTP fusion protein promoted DC maturation, enhanced the presentation of targeting antigens and efficiently induced HBcAg-specific CTL immune responses in vitro.
\end{abstract}

Correspondence to: Dr Guoqing Zang or Dr Zhenghao Tang, Department of Infectious Disease, Shanghai JiaoTong University Affiliated Sixth People's Hospital, 600 Yishuan Road, Shanghai 200233, P.R. China

E-mail: professorzangtg@126.com

E-mail: tzhhao@163.com

Key words: ubiquitin, cytoplasmic transduction peptide, hepatitis B virus core antigen, dendritic cells, antigen presentation, cytotoxic $\mathrm{T}$ lymphocytes

\section{Introduction}

Hepatitis B virus (HBV) infection remains a significant public health concern globally. Approximately 350 million individuals worldwide are chronically infected with HBV, and such infections may lead to the development of liver cirrhosis or hepatocellular carcinoma $(1,2)$. Although various types of antiviral drugs, including nucleotide/nucleotide analogue and interferon, have been used to eradicate this virus in recent years, no significant progress has been achieved (3). Increasing evidence has demonstrated that patients acutely infected with HBV usually develop marked, multispecific cytotoxic T lymphocyte (CTL) responses to the virus, whereas chronically infected individuals exhibit weak responses $(4,5)$. Therefore, the development of immunotherapeutic strategies to improve weak virus-specific T cell responses is critical.

Dendritic cells (DCs) are considered the most potent antigen-presenting cells (APCs); and as such, are able to initiate immune responses against invading pathogens (6) and have been observed to be responsible for the cross-presentation of antigens in vitro and in vivo as well as the stimulation of naïve cluster of differentiation (CD) $8^{+} \mathrm{T}$ cell proliferation and maturation (7). A previous study revealed that defective CTL responses may be attributed to impaired DC function (8). Therefore, promoting and improving the functions of DCs may comprise an efficient treatment strategy for persistent HBV infections.

Ubiquitin (Ub) is a highly conserved small regulatory protein (9), and a centrally significant component of the Ub-proteasome system (UPS), which attaches covalently to numerous cellular proteins through a highly regulated process $(10,11)$. Major histocompatibility complex (MHC) class-I antigen presentation is strictly dependent on the supply of appropriate peptides, mediated by the UPS, with which to efficiently prime $\mathrm{CD}^{+} \mathrm{T}$ cells and initiate an adaptive immune response $(12,13)$. The $\mathrm{HBV}$ core antigen $(\mathrm{HBcAg})$ is a highly immunogenic subviral particle that in natural and recombinant forms may induce marked immune responses characterized by acute T-cell activity (14). These hypotheses prompted the 
present study to investigate whether a Ub-modified $\mathrm{HBcAg}$ fusion protein was able to enter DCs and be presented by MHC class-I molecules in order to elicit robust CTL responses.

However, direct intracellular protein delivery is inhibited by the lipophilic nature of biological membranes (15). The cytoplasmic transduction peptide (CTP), which is derived from the protein transduction domain (PTD) of the human immunodeficiency virus-1 trans-activator of transcription protein, is a novel and deliberately designed transduction protein used to efficiently deliver biomolecules into the cytoplasm (16). Therefore, the properties of CTP provide an opportunity for antigens to enter DCs and thus be presented by MHC class I molecules.

In the present study, a prokaryotic expression vector for Ub-HBcAg-CTP (GGRRARRRRRR) was constructed and purified. Subsequently, the biological activity of the purified fusion protein was examined to determine whether it was able to be presented by MHC class-I molecules and consequently efficiently enhance $\mathrm{HBV}$-specific CTL responses in vitro.

\section{Materials and methods}

Animals and cell lines. The present study was approved by the Ethics Committee of Shanghai JiaoTong University Affiliated Sixth People's Hospital (Shanghai, China). Thirty BALB/c mice $\left(\mathrm{H}-2^{\mathrm{d}}\right)$, aged 6-8 weeks, were purchased from the Shanghai Experimental Animal Centre of the Chinese Academy of Sciences (Shanghai, China) and maintained in the Experimental Animal Center of the Shanghai Sixth People's Hospital under specific pathogen-free conditions (22-24 $4^{\circ}$; humidity 50-55\%; $12 \mathrm{~h}$ light/12 h dark cycle). The mice were pellet fed standard mouse food and given access to sterilized water. The mice were cared for and treated in accordance with the guidelines established by the Shanghai Public Health Service Policy on the Humane Care and Use of Laboratory Animals. After one week, $10 \mathrm{BALB} / \mathrm{c}$ mice were sacrificed by cervical dislocation following anesthesia with 3\% pentobarbital sodium (Sigma-Aldrich, St. Louis, MO, USA) via intramuscular injection (1:5 dilution; dose, $10 \mathrm{~g} / 0.1 \mathrm{ml}$ ).The bone marrow was collected from their femurs and tibiae, which was the source of the bone marrow cells. Briefly, the femurs and tibiae were removed from the mice and the surrounding muscle tissue was removed from the bones. The intact bones were placed in $70 \%$ ethanol for $5 \mathrm{~min}$, for disinfection, and were then washed with phosphate-buffered saline (PBS; Keygentec, Nanjing, China). Both ends were cut with scissors and the marrow was flushed with PBS. Clusters within the marrow suspension were dispersed by vigorous pipetting. The experiment was repeated three times.

HEK293T cells (Nanjing Medical University, Nanjing, China) were cultured in Dulbecco's modified Eagle's medium supplemented with $10 \%$ fetal bovine serum (FBS), $100 \mathrm{U} /$ $\mathrm{ml}$ penicillin and $100 \mu \mathrm{g} / \mathrm{ml}$ streptomycin (Invitrogen Life Technologies, Carlsbad, CA, USA), under humidified conditions with $5 \% \mathrm{CO}_{2}$ at $37^{\circ} \mathrm{C}$. The $\mathrm{H}-2^{\mathrm{d}}$ mastocytoma cell line P815/c (expressing the HBV core antigen) (Nanjing Medical University) was maintained in our lab (Department of Infectious Disease, Shanghai JiaoTong University Affiliated Sixth People's Hospital), and was cultured under the same conditions as the HEK293T cells.
Vector construction. The plasmid pcDNA3.1 (-)-Ub-HBcAg was constructed and maintained in our lab. The Ub-HBcAg cDNA sequence was generated via polymerase chain reaction (PCR) to obtain an 820 bp PCR product. The paired primer sequences were as follows: Forward, 5-AATGGATCCGGCGGCCGTCGTGCGCGTCGTCGTCGTCGTCGTATGGACAT TGACCCG-3' and reverse, 5'-CCCAAGCTTGCCACCTCTCAGG CGAAGG-3' (Sangon Biotech Co., Ltd., Shanghai, China). The underlined nucleotides represent the BamHI and HindIII sites, respectively. The Ub-HBcAg-CTP gene (Sangon Biotech Co., Ltd.) was inserted into the pMAL-c2X prokaryotic expression vector (Invitrogen Life Technologies) at the BamHI and HindIII (New England Biolabs, Ipswich, MA, USA) sites. The control genes (Ub-HBcAg and HBcAg-CTP) were also amplified via PCR and cloned separately into pMAL-c2X vectors. The aforementioned plasmids were further identified via restriction enzyme digestion and bidirectional DNA sequencing.

Protein expression, purification and western blotting. The recombinant plasmids were transformed into the Escherichia coli BL21 (DE3) bacterial strain (Keygentec) to induce the expression of the recombinant fusion proteins. Following being lysed by sonication (SM-650D; Shunma, Nanjing, China) and centrifuged (5415C; Thermo Fisher Scientific, Waltham, MA, USA) at $8,000 \mathrm{x}$ g for $5 \mathrm{~min}$ at $4^{\circ} \mathrm{C}$, the supernatants containing the $\mathrm{Ub}-\mathrm{HBcAg}-\mathrm{CTP}, \mathrm{Ub}-\mathrm{HBcAg}$ and $\mathrm{HBcAg-CTP}$ fusion proteins were purified using an amylose resin column (Polysciences, Inc., Eppelheim, Germany) according to the manufacturer's instructions and were evaluated via western blot analysis with an anti-HBcAg mouse monoclonal antibody (1:500 dilution; Abcam, Cambridge, UK) at $4{ }^{\circ} \mathrm{C}$ overnight, and a horseradish peroxidase-conjugated goat anti-mouse secondary monoclonal antibody (1:5,000 dilution; Wuhan Boster Biological Technology, Ltd., Wuhan, China) for $2 \mathrm{~h}$ at room temperature. The maltose binding protein-tag (Sangon Biotech Co., Ltd.) was ultimately cleaved by the Tobacco Etch Virus protease (Invitrogen Life Technologies). All proteins were stored at $4^{\circ} \mathrm{C}$ until use.

DC generation. DCs were generated according to a previously published method with certain modifications (17). Briefly, bone marrow cells from the femurs and tibiae of the mice were collected and cultured at a density of $2 \times 10^{6}$ cells $/ \mathrm{ml}$ in RPMI 1640 medium (Hyclone Biocehmical Product Co., Ltd., Beijing, China) supplemented with $10 \%$ FBS, $20 \mathrm{ng} / \mathrm{ml}$ recombinant mouse granulocyte-monocyte colony stimulating factor (rmGM-CSF; Peprotech EC Ltd., London, UK) and $10 \mathrm{ng} / \mathrm{ml}$ recombinant mouse interleukin 4 (rmIL-4; Peprotech EC Ltd.). Following a two day incubation, the adherent cells were divided into five groups, and four of the groups were cultured for an additional $72 \mathrm{~h}$ in the presence of Ub-HBcAg-CTP, Ub-HBcAg, HBcAg-CTP and HBcAg (Abcam; all concentrations $20 \mu \mathrm{g} / \mathrm{ml}$ ), respectively. All groups were treated with lipopolysaccharide (20 ng/ml; Sigma-Aldrich). On day eight, the non-adherent and loosely adherent cells were harvested as DCs.

DC morphology, intracellular localization and western blot analysis. The day five and day eight DCs were observed via 
scanning (Quanta 450) and transmission (Tecnai 12) electron microscopy (FEI Company, Eindhobven, Netherlands). The samples were treated according to the standard experimental methods (18). The day five DCs were cocultured with the aforementioned proteins at a concentration of $20 \mu \mathrm{g} / \mathrm{ml}$ for $24 \mathrm{~h}$. Following washing with PBS, the cells were fixed in $100 \%$ pre-chilled methanol (Keygentec). Following an additional three washes with PBS, the cells were permeabilized with $0.3 \%$ Triton X-100 (Keygentec) and blocked with $10 \%$ normal goat serum (Wuhan Boster Biological Technology, Ltd.). The cells were subsequently incubated overnight with an anti-HBcAg mAb (1:500 dilution) at $4^{\circ} \mathrm{C}$. The cells were then further incubated with goat anti-mouse fluorescein isothiocyanate (FITC)-Immunoglobulin G (Wuhan Boster Biological Technology, Ltd.) for $1 \mathrm{~h}$ at room temperature. Following washing with PBS and DAPI-staining (Keygentec), the cells were visualized with a LSM 510 laser scanning confocal microscope (Carl Zeiss, Oberkochen, Germany) and analyzed using LSM image examiner software (Carl Zeiss). The mean fluorescence intensity (MFI) of the entire view was calculated from five sites (the four corners and center of each section).

Day five DCs, which were treated as described above, were also cocultured with or without the specific proteasome inhibitor MG-132 (10 $\mu \mathrm{mol}$; Sigma-Aldrich). Following $24 \mathrm{~h}$ of incubation, the DCs were harvested to analyze the level of $\mathrm{HBcAg}$ via western blotting.

Western blot analysis. Following a $24 \mathrm{~h}$ incubation the DCs were harvested and washed twice with PBS. The cells were then gently dispersed into a single-cell suspension and homogenized using radioimmunoprecipitation assay lysis buffer (Keygentec). Protein concentrations were determined using Pierce Bicinchoninic Acid Protein Assay Reagent kit (Pierce Biotechnology, Inc., Rockford, IL, USA). The homogenates were diluted to the desired protein concentration using $2 \mathrm{X}$ SDS-PAGE loading buffer (Invitrogen Life Technologies). The samples were then boiled and loaded onto polyacrylamide mini-gels (Invitrogen Life Technologies) for electrophoresis. The protein samples were then transferred to polyvinylidene fluoride membranes (EMD Millipore, Bedford, MA, USA) using semi-dry apparatus. The membranes were then incubated with $\mathrm{HBcAg}$ monoclonal human anti-mouse antibody at $4^{\circ} \mathrm{C}$ overnight, followed by an incubation with horseradish peroxidase-conjugated goat anti-mouse immunoglobulin $\mathrm{G}$ secondary antibody at room temperature for $2 \mathrm{~h}$. GAPDH was used as the control (GAPDH antibody, 1:1,000, $4^{\circ} \mathrm{C}$ overnight; Santa Cruz Biotechnology, Inc., Santa Cruz, CA, USA). Image-Pro Plus (version, 6.0; Media Cybernetics, Inc, Bethesda, MD, USA) was used to visualize and quantify the blots. Gray value was used to compare the differences between the groups. Gray value=gray value of $\mathrm{HBcAg} / \mathrm{gray}$ value of GAPDH.

IL-12p70 production, DC immunophenotypic analysis and $T$ cell proliferation. Day 5 DCs were cocultured with the aforementioned proteins for $72 \mathrm{~h}$, and the IL-12p70 concentrations in the supernatants were measured using a standard sandwich ELISA kit (R\&D Systems, Minneapolis, MN, USA) according to the manufacturer's instructions. The concentrations were calculated and expressed as $\mathrm{pg} / \mathrm{ml}$. The surface molecules of day eight DCs were analyzed following incubation with phycoerythrin (PE)-labeled monoclonal antibodies against mouse CD11c (1:50 dilution), CD80 (1:50 dilution), CD83 (1:100 dilution), CD86 (1:100 dilution) and MHC class-I (1:40 dilution) (eBioscience, San Diego, CA, USA) at $4^{\circ} \mathrm{C}$ in the dark for $30 \mathrm{~min}$. Fluorescence analyses were performed on a COULTER EPICS XL Flow Cytometer (Beckman Coulter, Miami, FL, USA) using Expo32-ADC software (Beckman Coulter).

Day five DCs were cocultured with Ub-HBcAg-CTP, Ub-HBcAg, HBcAg-CTP and HBcAg (20 $\mu \mathrm{g} / \mathrm{ml}$; Abcam). After $72 \mathrm{~h}$, day eight DCs were pretreated with $25 \mu \mathrm{g} / \mathrm{ml}$ mitomycin C (Sigma-Aldrich) for $30 \mathrm{~min}$. T cells were sorted from splenocytes of allogenenic naïve mice using nylon wool columns (Polysciences. Inc.) and grown as responder cells in coculture with DCs at various responder/stimulator (T cell/DC) ratios (5:1, 10:1 and 20:1). The cells were incubated in a final volume of $100 \mu \mathrm{l}$ for $96 \mathrm{~h}$, during which $10 \mu \mathrm{l}$ Cell Counting Kit-8 solution (Dojindo Laboratories, Kumamoto, Japan) was added for $4 \mathrm{~h}$. The absorbance values of the cultures were read at a wavelength of $450 \mathrm{~nm}$ (Multiskan Ascent; Thermo Fisher Scientific).

Intracellular cytokine analysis of proliferative $T$ cells. Proliferative T cells were stimulated for $6 \mathrm{~h}$ in the presence of $25 \mu \mathrm{g} / \mathrm{ml}$ phorbol 12-myristate 13-acetate (Sigma-Aldrich), $1 \mu \mathrm{g} / \mathrm{ml}$ ionomycin (Sigma-Aldrich) and $1.7 \mu \mathrm{g} / \mathrm{ml}$ monensin (Sigma-Aldrich) (19). The cells were subsequently stained with a PE-Cy5-conjugated anti-CD3 mAb (eBioscience) and a FITC-conjugated anti-CD $8 \alpha \mathrm{mAb}$ (eBioscience) for $15 \mathrm{~min}$ at room temperature. Following fixation and permeabilization using the Fix/Perm reagents $\mathrm{A}$ and $\mathrm{B}$ (BD Biosciences, San Jose, CA, USA) for 15 and 5 min respectively, the cells were incubated with a PE-labeled anti-interferon (IFN)- $\gamma$ $\mathrm{mAb}$ (eBioscience) for $30 \mathrm{~min}$. Fluorescence analyses were performed on a COULTER EPICS XL flow cytometer (Beckman Coulter) with Expo 32-Advanced Digital Compensation software $\left(\right.$ Navios $^{\mathrm{TM}}$ Cytometer, version 1.0; Beckman Coulter).

Cytokine secretion and CTL assay. T cells were cocultured with mature DCs in a humidified atmosphere containing $5 \%$ $\mathrm{CO}_{2}$ at $37^{\circ} \mathrm{C}$ for four days at a $\mathrm{T}$ cell/DC ratio of 10:1. The concentrations of various cytokines (IFN- $\gamma$ and IL-2) in the supernatants were measured using mouse cytokine ELISA kits (R\&D Systems). The concentrations were expressed as pg/ml.

The P815/c cells were seeded as the target cells, and previously stimulated $\mathrm{T}$ cells were used as the effector cells. The $\mathrm{T}$ cells were cocultured with the $\mathrm{P} 815 / \mathrm{c}$ cells at effector/target ratios of $5: 1,10: 1$ and $20: 1$ for $4 \mathrm{~h}$ at $37^{\circ} \mathrm{C}$ in a humidified atmosphere containing $5 \% \mathrm{CO}_{2}$. $\mathrm{HBcAg}$-specific CTL activity was measured using a lactate dehydrogenase release assay (CytoTox 96 ${ }^{\circledR}$ non-radioactive cytotoxicity kit; Promega Corporation, Madison, WI, USA), according to the manufacturer's instructions. The absorbance values were recorded at a wavelength of $490 \mathrm{~nm}$ (Multiskan Ascent). The percentage cytotoxicity was calculated as follows: [(experimental release - effector spontaneous release - target spontaneous release) / (target maximum release - target spontaneous release)] x $100(20)$. 
A

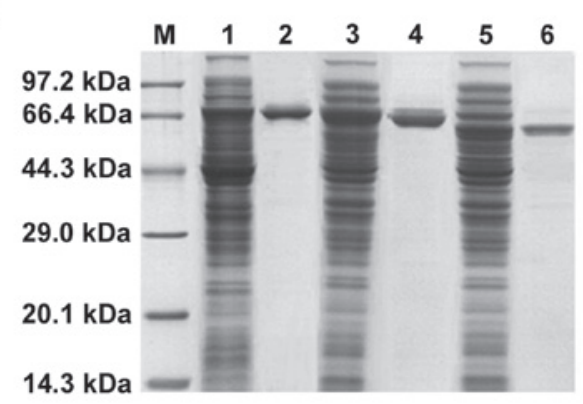

B

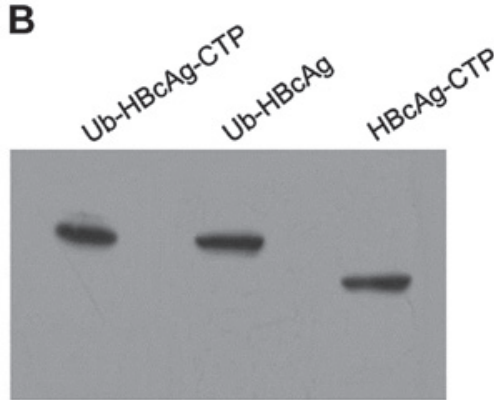

C
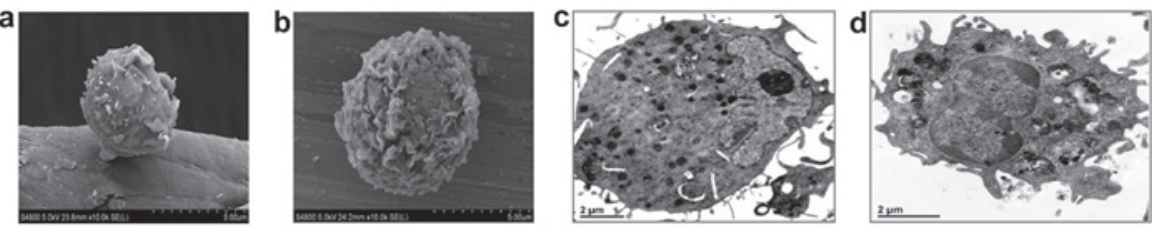

Figure 1. Fusion protein expression, purification, western blot analysis and DC morphology. (A) Expression and purification of fusion proteins by SDS-PAGE. Lanes: M, molecular weight standards; 1 and 2, expression and purification of MBP-Ub-HBcAg-CTP recombinant protein, respectively; 3 and 4, expression and purification of MBP-Ub-HBcAg recombinant protein, respectively; 5 and 6, expression and purification of MBP-HBcAg-CTP recombinant protein, respectively. (B) Purified fusion proteins were analyzed via western blotting using anti-HBcAg monoclonal antibody. (C) Ultrastructures of day five and day eight DCs were observed using (a and b) scanning electron microscopy (magnification, $\mathrm{x} 5,000)$ and (c and d) transmission electron microscopy (magnification, x20,000), respectively. HBcAg, hepatitis B virus core antigen; DC, dendritic cell; CTP, cytoplasmic transduction peptide; MBP, maltose binding protein; Ub, ubiquitin.

Statistical analysis. Each value in the present study was obtained from a minimum of three independent experiments and data are expressed as the mean \pm standard deviation. The differences between groups were determined using one-way analysis of variance. Statistical data analyses were performed with SPSS 16.0 software (SPSS, Inc., Chicago, IL, USA). $\mathrm{P}<0.05$ was considered to indicate a statistically significant difference.

\section{Results}

Fusion protein expression, purification and western blotting. The Ub-HBcAg-CTP, Ub-HBcAg and HBcAg-CTP fusion gene constructs were 813,780 and 585 bp in size, respectively, as determined by PCR amplification. All the target prokaryotic expression plasmids were successfully constructed and identified by restriction endonuclease analysis and bidirectional DNA sequencing (data not shown). The purified fusion proteins were identified to be $\sim 80 \%$ pure via SDS-PAGE analysis (Fig. 1A) and the products were further confirmed by western blot analysis (Fig. 1B).

DC maturation, cytoplasmic localization and western blotting. On the DC surfaces, increased numbers of ruffles and rough dendrites were observed by scanning electron microscopy. Similarly, the morphological characteristics of mature DCs were detected by transmission electron microscopy, and identified larger nuclei, as well as increased numbers of mitochondria and ribosomes (Fig. 1C).

Immunofluorescent staining and confocal microscopy were used to characterize the intracellular localization of the various proteins in DCs. The HBcAg protein was identified in the cytoplasm of DCs treated with Ub-HBcAg-CTP and $\mathrm{HBcAg-CTP}$ via the presence of green fluorescence (FITC-labeled HBcAG) (Fig. 2A). This fluorescence was clearly distinguished from the nuclear-specific counterstain, DAPI. Green fluorescence was observed to aggregate primarily on the DC cytomembranes in the Ub-HBcAg and HBcAg-treated groups (Fig. 2A), demonstrating the potent transduction potential and cytoplasmic localization mediated by the CTP peptide. The green fluorescence intensity detected in the Ub-HBcAg-CTP group was weaker than that in the HBcAg-CTP group, indirectly demonstrating the lower $\mathrm{HBcAg}$ levels in the former group (Fig. 2B). Furthermore, the levels of $\mathrm{HBcAg}$ were detected in the Ub-HBcAg-CTP and $\mathrm{HBcAg-CTP}$ groups using western blot analysis to determine the rates of intracellular degradation. As expected, $\mathrm{HBcAg}$ levels in Ub-HBcAg-CTP-group were lower than those in the $\mathrm{HBcAg-CTP-group,} \mathrm{but} \mathrm{recovered} \mathrm{to} \mathrm{the} \mathrm{same} \mathrm{level} \mathrm{as} \mathrm{those} \mathrm{of}$ $\mathrm{HBcAg}$ following the addition of MG-132 (Fig. 2C).

Ub-HBcAg-CTP leads to increased IL-12 production and surface molecule expression. IL-12 is considered a vital cytokine, produced by DCs, and is a key cytokine involved in the induction of Type-1 immune responses (21). The IL-12p70 concentrations were assayed in the various protein-treated groups. The results demonstrated that DCs cocultured with Ub-HBcAg-CTP secreted significantly greater quantities of IL-12p70 $(84.38 \pm 9.625 \mathrm{pg} / \mathrm{ml})$ than those of the other groups $(\mathrm{P}<0.05$; Fig. 3B). However, no differences were identified among the other groups. Additionally, following treatment, the quantity of DCs $\left(\mathrm{CD} 11 \mathrm{c}^{+}\right)$in the culture was $\sim 85 \%$ (flow cytometric analysis) and the surface molecules CD80, CD83, CD86 and MHC class-I were highly expressed on the Ub-HBcAg-CTP-treated DCs (Fig. 3A).

T-cell proliferation. T cells were cocultured with DCs, which had been pulsed with various proteins in order to analyze their T-cell proliferation capacities. It was identified that the T-cell proliferation capacity was markedly higher in the 
A
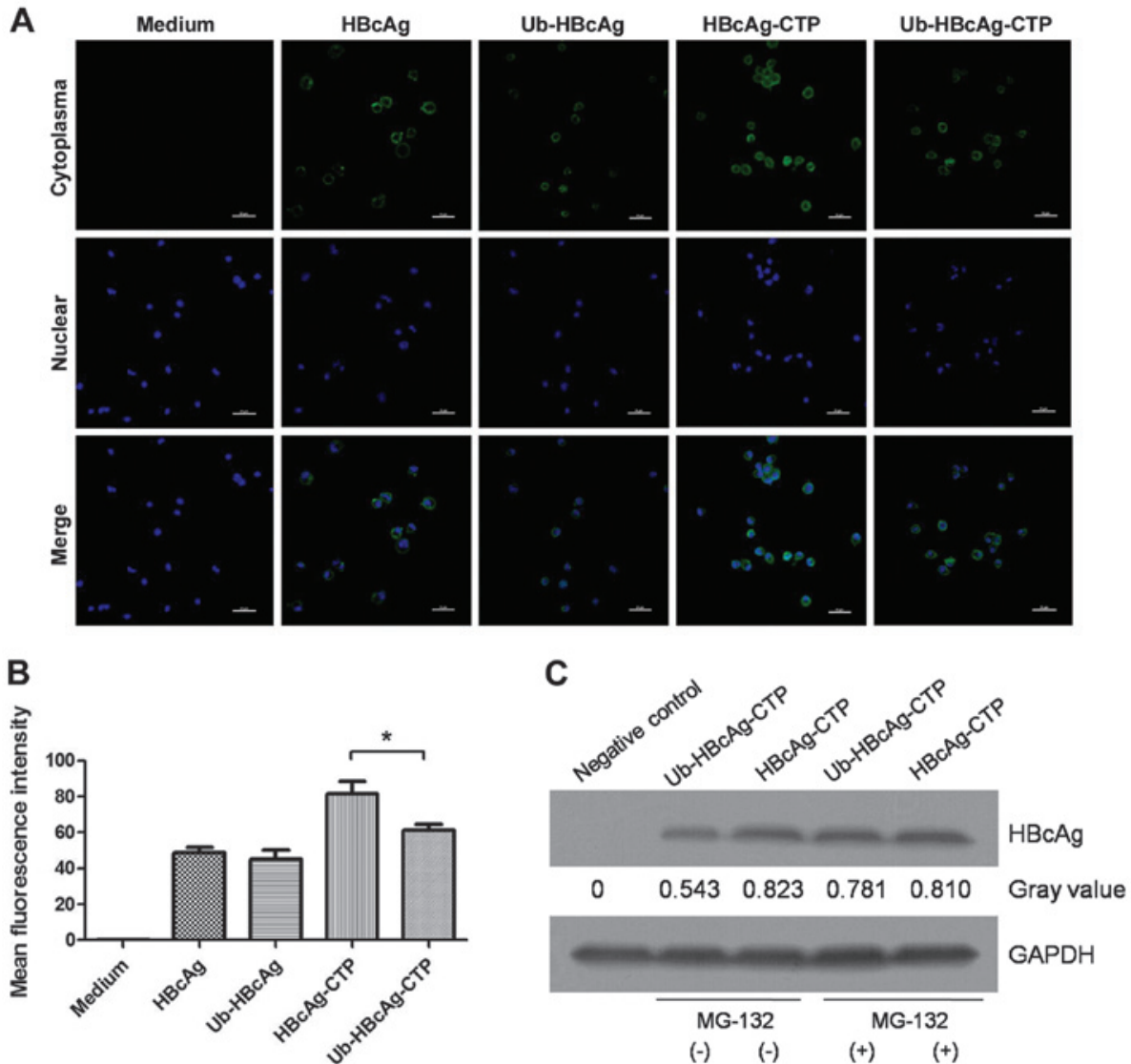

Figure 2. Intracellular localization and western blot analysis. (A) Confocal microscopic analysis of DCs treated with various proteins. Nuclei were stained with DAPI (blue). Fluorescein isothiocyanate-labeled HBcAg (green fluorescence) was detected mainly in the cytoplasm (magnification, x200). (B) Analysis of mean fluorescence intensity was performed in the cytoplasm of DCs treated with various fusion proteins via confocal microscopy. Data is expressed as the mean \pm standard deviation. ${ }^{\mathrm{P}}<0.05$. (C) Detection of the levels of HBcAg in the cytoplasm of DCs treated with Ub-HBcAg-CTP and HBcAg-CTP. MG-132 (10 $\mu \mathrm{mol})$ was added for $24 \mathrm{~h}$. Cell lysates were analyzed using western blotting. Relative expression of $\mathrm{HBcAg}$ was calculated using a gray value. HBcAg, hepatitis B virus core antigen; DC, dendritic cell; CTP, cytoplasmic transduction peptide; MBP, maltose binding protein; Ub, ubiquitin.

Ub-HBcAg-CTP group (P<0.05; Fig. 3C), and was enhanced by lower $\mathrm{T}$ cell//DC ratios.

Ub-HBcAg-CTP leads to increased IFN- $\gamma$ production by $C D 8^{+}$T cells in vitro. The levels of $\mathrm{CD}^{+} \mathrm{T}$ cell-produced IFN- $\gamma$ were measured by intracellular staining and flow cytometry. The cells were stained with an anti-CD3 mAb to identify $\mathrm{T}$ cells within the cell populations, and the samples were subsequently subjected to intracellular staining. As shown in Fig. 4A, the percentage of specific IFN $-\gamma^{+} \mathrm{CD}^{+} \mathrm{T}$ cells was greater in the Ub-HBcAg-CTP group than in the other groups $(\mathrm{P}<0.05)$, suggesting that Ub-HBcAg-CTP-treated DCs may enhance the generation of specific CTLs.

Ub-HBcAg-CTP-induces the enhancement of cytokine production and CTL activity. Subsequently, the levels of IFN- $\gamma$ and IL-2 secreted by T cells were measured. The Ub-HBcAg-CTP group produced higher levels of IFN- $\gamma(348.8 \pm 24.78 \mathrm{pg} / \mathrm{ml})$ and IL-2 $(476.5 \pm 20.81 \mathrm{pg} / \mathrm{ml})$ than those in the other groups $(\mathrm{P}<0.05$, Fig. 4B). Clearer CTL responses were detected at differing effector/target ratios in the Ub-HBcAg-CTP group, compared with those in the other groups (Fig. 4C). These results suggested that $\mathrm{Ub}-\mathrm{HBcAg-CTP}$ may induce marked specific CTL responses, which was consistent with the high level of IFN- $\gamma$ expressed in $\mathrm{CD}^{+} \mathrm{T}$ cells.

\section{Discussion}

Various immunotherapeutic strategies have been developed to eliminate $\mathrm{HBV}$; however, these strategies have not had a substantial impact (22). The host antiviral immune response, particularly CTL activity, to HBV-antigens has been established as the main determinant in the processes of viral replication and clearance $(23,24)$. Therefore, the induction of $\mathrm{HBV}$-specific $\mathrm{CD}^{+} \mathrm{T}$ cells is a current goal in the development of effective immune-based therapeutic interventions for the treatment of HBV infection, intended to enhance antigen presentation in order to induce broad CTL responses.

$\mathrm{CD}^{+} \mathrm{T}$ cell priming requires the direct and/or cross-presentation of antigenic peptides on MHC class I molecules by APCs (25). DCs are among the most potent APCs and possess a unique capacity to interact with naïve $\mathrm{T}$ cells and consequently induce immune responses (26). Patients with chronic $\mathrm{HBV}$ infections generally exist in an immune-comprised state, comprising immune tolerance with impaired DC function $(27,28)$. Therefore, efforts should be made to enhance the antigen presenting capacities of DCs.

CTP, which is derived from PTD, was verified to facilitate specific cytoplasmic localization and possess potent membrane transduction potential (16). Accordingly, cytoplasmic functional molecules may be more efficiently targeted 

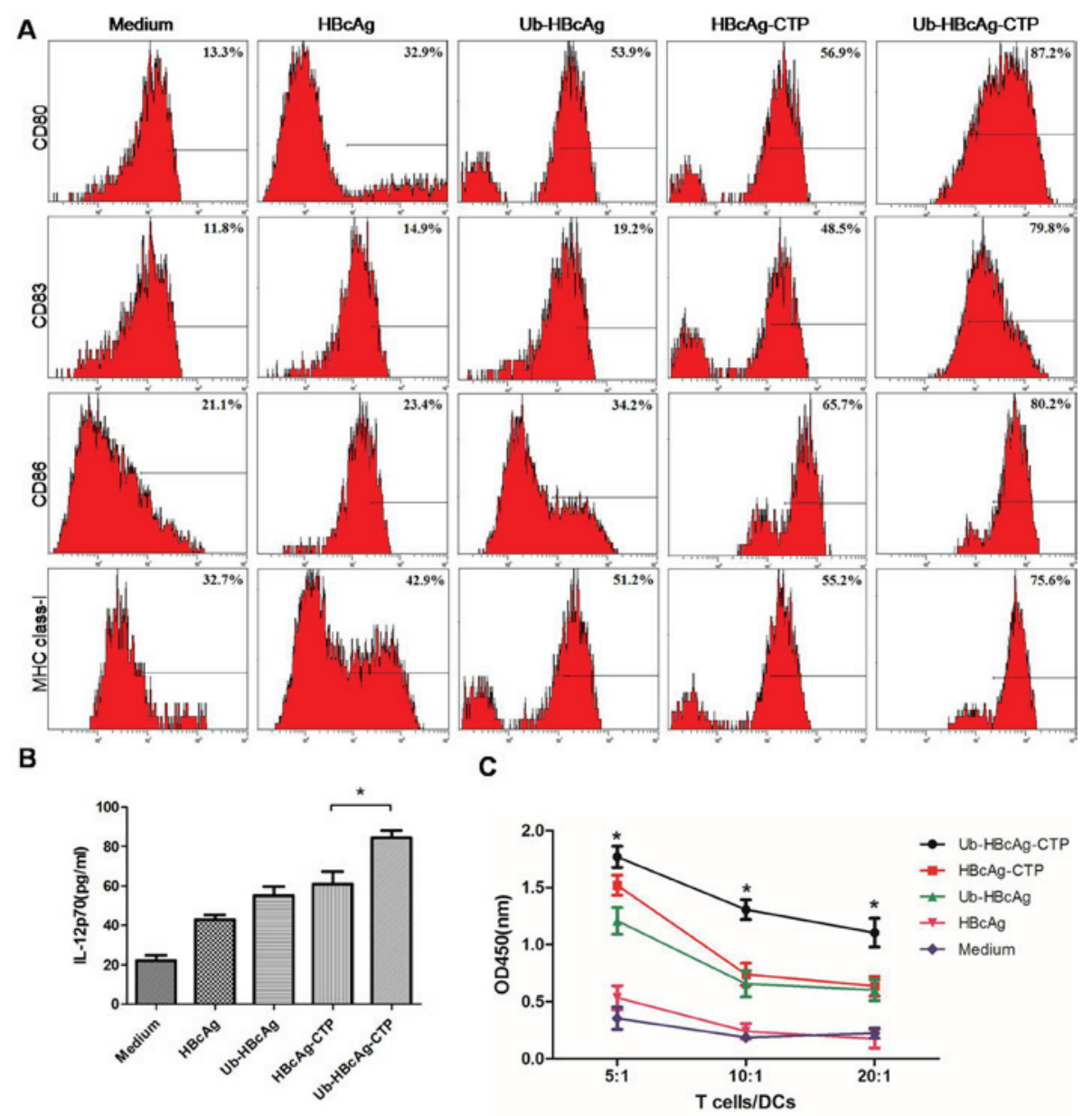

Figure 3. DC immunophenotypic analysis, detection of IL-12p70 production and T-cell proliferation. (A) Surface molecules on DCs (CD80, CD83, CD86, and major histocompatibility complex class-I) were detected using flow cytometry. Surface molecule expression was significantly higher in the Ub-HBcAg-CTP group than in the other groups. ${ }^{*} \mathrm{P}<0.05,{ }^{* * *} \mathrm{P}<0.01$. (B) IL-12p70 concentrations in supernatants of DCs were measured via ELISA. Data is expressed as the mean \pm SD. ${ }^{*} \mathrm{P}<0.05$ vs. HBcAg-CTP group. (C) T-cell proliferation capacities at various responder/stimulator (T cell/DC) ratios. Data is expressed as the mean $\pm \mathrm{SD} .{ }^{*} \mathrm{P}<0.05,{ }^{* *} \mathrm{P}<0.01$ vs. HBcAg-CTP group. HBcAg, hepatitis B virus core antigen; DC, dendritic cell; CTP, cytoplasmic transduction peptide; MBP, maltose binding protein; Ub, ubiquitin; SD, standard deviation; IL, interleukin; $\mathrm{CD}$, cluster of differentiation; OD, optical density.

A

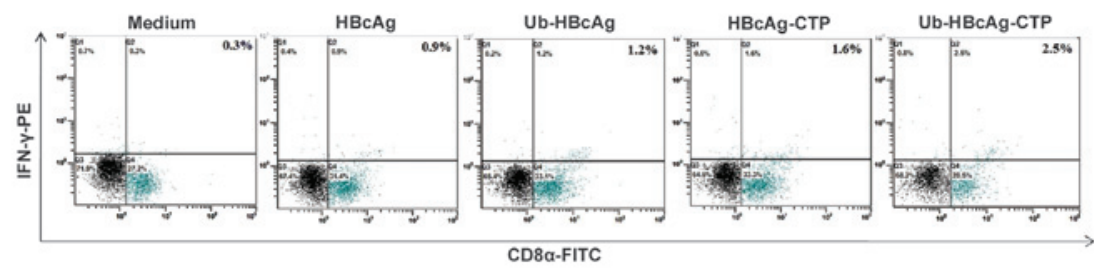

B

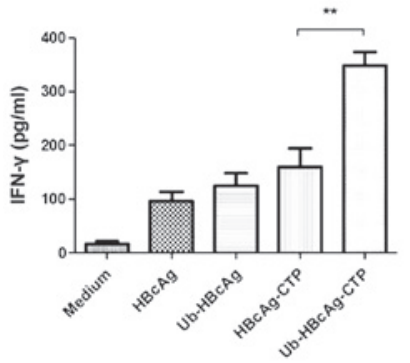

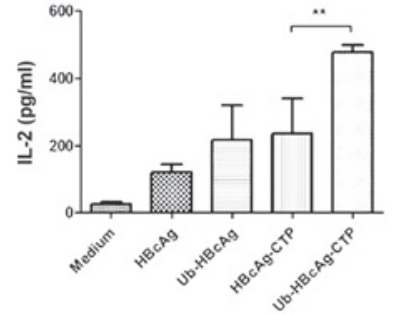

C ${ }_{80} \rightarrow$ Ub-HBCAg-CTP

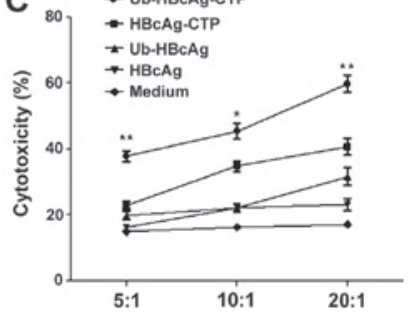

Figure 4. Intracellular cytokine analysis of proliferative $\mathrm{T}$ cells, cytokine production and CTL assay. (A) Specific IFN- $\gamma^{+} \mathrm{CD} 8^{+} \mathrm{T}$ cells were detected by flow cytometry. (B) Levels of IFN- $\gamma$ and IL-2 in the supernatants of proliferative T cells stimulated by various protein-treated DCs. Data is expressed as the mean \pm standard deviation. ${ }^{*} \mathrm{P}<0.05$. (C) Specific CTL activity was measured by lactate dehydrogenase release assay. The proliferative $\mathrm{T}$ cells were incubated with target cells at various effector/target ratios (5:1, 10:1 and 20:1). CTL activity was indicated as the mean percentage of specific lysis (mean \pm standard deviation). "P<0.05, ${ }^{* *} \mathrm{P}<0.01$, compared with control. CTL, cytotoxic T lymphocytes; IFN- $\gamma$, interferon- $\gamma$; IL, interleukin; CD, cluster of differentiation; DC, dendritic cell; HBcAg, hepatitis B virus core antigen; CTP, cytoplasmic transduction peptide; Ub, ubiquitin; FITC, fluorescein isothiocyanate. 
by CTP-mediated delivery $(16,29)$. In the present study, a $\mathrm{Ub}-\mathrm{HBcAg-CTP}$-expressing vector was constructed in order to achieve increased transduction into cells. Following transduction, the Ub-HBcAg-CTP fusion protein was detected via confocal microscopy, in order to characterize the intracellular localization. It was revealed that CTP-containing fusion proteins remained primarily in the cytoplasm of DCs, whereas proteins without CTP were detected primarily on the cell surface. This result confirmed that target proteins were able to be transported efficiently into the DC cytoplasm via CTP, thus providing a basis for the further verification of protein immunogenicity.

$\mathrm{Ub}$ is a well-conserved and ubiquitously expressed protein that is conjugated to target proteins (30). It is best known for its role as a marker for protein destruction by the UPS (31). In the present study, the MFI of Ub-HBcAg-CTP-transduced DCs was weaker than that of the HBcAg-CTP group, thus indirectly demonstrating the degradation of $\mathrm{HBcAg}$. Furthermore, $\mathrm{HBcAg}$ was quantitatively measured by western blot analysis. It was identified that the Ub-tagged $\mathrm{HBcAg}$ protein was at a lower level in the absence of MG-132 (specific proteasome inhibitor), indicating rapid recognition of the fusion protein by the UPS, leading to $\mathrm{HBcAg}$ degradation. Ub-mediated antigen processing has been widely used to enhance immune responses in infectious disease and cancer studies $(32,33)$. A previous study confirmed that Ub-fused proteins may improve CTL activity by promoting the introduction of the encoded protein into the MHC class I pathway (34). HBcAg, which possesses unique immunological features, elicits prominent immune responses during chronic $\mathrm{HBV}$ infection (35). Therefore, a Ub-modified $\mathrm{HBcAg}$ gene was amplified.

In the present study, the immunomodulatory effects of these proteins on bone marrow-derived DC (BMDC) development in vitro was investigated. It was identified that Ub-HBcAg-CTP-treated BMDCs expressed higher levels of surface molecules. Additionally, the associated mature DCs secreted higher levels of IL-12 in response to various intracellular pathogenic infections. This response has a key role in the initiation of specific $\mathrm{T}$ cell-mediated immune responses and the promotion of T-helper (Th) 1 cell activation and differentiation (21). Accordingly, IL-12p70 production was evaluated. As desired, the DC production of IL-12p70 was markedly increased in response to $\mathrm{Ub}-\mathrm{HBcAg}-\mathrm{CTP}$ treatment, compared with that in the other treatment groups. The DC morphology in the Ub-HBcAg-CTP group was also observed to include numerous mitochondria, as well as increased quantities of ribosomes and rough endoplasmic reticulum. The results demonstrated that the Ub-modified $\mathrm{HBcAg}$ protein may facilitate DC maturation and increase MHC class-I expression.

Th1 cells are known to produce large quantities of Type- 1 cytokines, including IFN- $\gamma$ and IL-2, whereas Th2 cells produce large quantities of Type- 2 cytokines, including IL-4 and IL-10 (36). In the current study, the secretion of Type-1 cytokines (IFN- $\gamma$ and IL-2) was demonstrated to be significantly increased in the Ub-HBcAg-CTP group, which indicated that Ub-HBcAg-CTP-pulsed DCs had a tendency to promote Th1 polarization and activate cell-mediated immunity. In addition, in order to determine whether this maturation sufficiently modulated $\mathrm{CD}^{+} \mathrm{T}$ cells, the production of IFN- $\gamma$ was measured via intracellular cytokine staining. The cells in the Ub-HBcAg-CTP group were found to induce a greater quantity of IFN- $\gamma^{+} \mathrm{CD} 8^{+} \mathrm{T}$ cells and more robust specific CTL activity. The specific cytotoxic response assay confirmed the enhancing effect of Ub-HBcAg-CTP on specific CTL responses. The results demonstrated that $\mathrm{Ub}-\mathrm{HBcAg-CTP}$ fusion proteins may enhance the capacity of T cells to proliferate, secrete cytokines and develop into CTLs in vitro. Based on these findings, it is possible that the role of the Ub-HBcAg-CTP fusion protein in the induction of specific CTLs may be attributed to the fact that UPS-degraded antigenic peptides are presented by cell-surface-expressed MHC class-I molecules and are, therefore, specifically recognized by CTLs.

In conclusion, the present study demonstrated that $\mathrm{Ub}-\mathrm{HBcAg}$-CTP fusion proteins do not only increase the expression of MHC class-I molecules, but also enhance the presentation of antigenic peptides and elicit robust $\mathrm{HBcAg}$-specific CTL immune responses in vitro. Although the data presented in the present study may not be fully translatable to studies in humans, these findings may suggest a potential therapeutic strategy for chronically infected HBV patients.

\section{Acknowledgements}

The present study was supported by grants from the National Natural Science Foundation of China (grant no. 81270502) and the Natural Science Foundation of Shanghai (grant no. 11ZR1427100).

\section{References}

1. Ganem D and Prince AM: Hepatitis B virus infection - natural history and clinical consequences. New Engl J Med 350: 1118-1129, 2004.

2. Shepard CW, Simard EP, Finelli L, Fiore AE and Bell BP: Hepatitis B virus infection: epidemiology and vaccination. Epidemiol Rev 28: 112-125, 2006.

3. Raney AK, Hamatake RK and Hong Z: Agents in clinical development for the treatment of chronic hepatitis B. Expert Opin Investig Drugs 12: 1281-1295, 2003.

4. Rehermann B: Immune responses in hepatitis B virus infection. Semin Liver Dis 23: 21-38, 2003.

5. Kakimi K, Isogawa M, Chung J, Sette A and Chisari FV: Immunogenicity and tolerogenicity of hepatitis B virus structural and nonstructural proteins: implications for immunotherapy of persistent viral infections. J Virol 76: 8609-8620, 2002.

6. Steinman RM and Hemmi H: Dendritic cells: translating innate to adaptive immunity. Curr Top Microbiol Immunol 311: 17-58, 2006.

7. Pozzi LA, Maciaszek JW and Rock KL: Both dendritic cells and macrophages can stimulate naive CD8 $\mathrm{T}$ cells in vivo to proliferate, develop effector function, and differentiate into memory cells. J Immunol 175: 2071-2081, 2005.

8. Almand B, Resser JR, Lindman B, et al: Clinical significance of defective dendritic cell differentiation in cancer. Clin Cancer Res 6: 1755-1766, 2000.

9. Glickman $\mathrm{MH}$ and Ciechanover A: The ubiquitin-proteasome proteolytic pathway: destruction for the sake of construction. Physiol Rev 82: 373-428, 2002.

10. Shabek $\mathrm{N}$ and Ciechanover A: Degradation of ubiquitin: the fate of the cellular reaper. Cell Cycle 9: 523-530, 2010.

11. Hershko A and Ciechanover A: The ubiquitin system. Annu Rev Biochem 67: 425-479, 1998.

12. Warnatsch A, Bergann T and Krüger E: Oxidation matters: the ubiquitin proteasome system connects innate immune mechanisms with MHC class I antigen presentation. Mol Immunol 55: 106-109, 2013.

13. Burr ML, Boname JM and Lehner PJ: Studying ubiquitination of MHC class I molecules. Methods Mol Biol 960: 109-125, 2013. 
14. Schödel F, Moriarty AM, Peterson DL, et al: The position of heterologous epitopes inserted in hepatitis B virus core particles determines their immunogenicity. J Virol 66: 106-114, 1992.

15. Singh TR, Garland MJ, Cassidy CM, et al: Microporation techniques for enhanced delivery of therapeutic agents. Recent Pat Drug Deliv Formul 4: 1-17, 2010.

16. Kim D, Jeon C, Kim JH, et al: Cytoplasmic transduction peptide (CTP): new approach for the delivery of biomolecules into cytoplasm in vitro and in vivo. Exp Cell Res 312: 1277-1288, 2006.

17. Huang Y, Chen Z, Jia H, Wu W, Zhong S and Zhou C: Induction of Tc1 response and enhanced cytotoxic T lymphocyte activity in mice by dendritic cells transduced with adenovirus expressing HBsAg. Clin Immunol 119: 280-290, 2006.

18. Tatu RF, Anuşca DN, Groza SŞ, et al: Morphological and functional characterization of femoral head drilling-derived mesenchymal stem cells. Rom J Morphol Embryol 55: 1415-1422, 2014.

19. Crawford TQ, Ndhlovu LC, Tan A, et al: HIV-1 infection abrogates $\mathrm{CD} 8+\mathrm{T}$ cell mitogen-activated protein kinase signaling responses. J Virol 85: 12343-12350, 2011.

20. Chen JH, Yu YS, Chen XH, Liu HH, Zang GQ and Tang ZH: Enhancement of CTLs induced by DCs loaded with ubiquitinated hepatitis B virus core antigen. World J Gastroenterol 18: $1319-1327,2012$

21. Giermasz AS, Urban JA, Nakamura Y, et al: Type-1 polarized dendritic cells primed for high IL-12 production show enhanced activity as cancer vaccines. Cancer Immunol Immunother 58: 1329-1336, 2009.

22. Wang L, Zou ZQ, Liu CX and Liu XZ: Immunotherapeutic interventions in chronic hepatitis B virus infection: a review. J Immunol Methods 407: 1-8, 2014

23. Tsai SL, Sheen IS, Chien RN, et al: Activation of Th1 immunity is a common immune mechanism for the successful treatment of hepatitis B and C: tetramer assay and therapeutic implications. J Biomed Sci 10: 120-135, 2003.

24. Phillips S, Chokshi S, Riva A, Evans A, Williams R and Naoumov NV: CD8(+) T cell control of hepatitis B virus replication: direct comparison between cytolytic and noncytolytic functions. J Immunol 184: 287-295, 2010.

25. Schliehe C, Bitzer A, van den Broek M and Groettrup M: Stable antigen is most effective for eliciting $\mathrm{CD} 8^{+} \mathrm{T}$-cell responses after DNA vaccination and infection with recombinant vaccinia virus in vivo. J Virol 86: 9782-9793, 2012.
26. Chiang CL, Balint K, Coukos G and Kandalaft LE: Potential approaches for more successful dendritic cell-based immunotherapy. Expert Opin Biol Ther Jan 2, 2015 (Epub ahead of print).

27. Tavakoli S, Mederacke I, Herzog-Hauff S, et al: Peripheral blood dendritic cells are phenotypically and functionally intact in chronic hepatitis B virus (HBV) infection. Clin Exp Immunol 151: 61-70, 2008.

28. Op den Brouw ML, Binda RS, van Roosmalen MH, et al: Hepatitis B virus surface antigen impairs myeloid dendritic cell function: a possible immune escape mechanism of hepatitis B virus. Immunology 126: 280-289, 2009.

29. Chen HZ, Wu CP, Chao YC and Liu CY: Membrane penetrating peptides greatly enhance baculovirus transduction efficiency into mammalian cells. Biochem Biophys Res Commun 405: 297-302, 2011.

30. Schaefer A, Nethe M and Hordijk PL: Ubiquitin links to cytoskeletal dynamics, cell adhesion and migration. Biochem J 442: 13-25, 2012.

31. Gao $\mathrm{G}$ and Luo H: The ubiquitin-proteasome pathway in viral infections. Can J Physiol Pharmacol 84: 5-14, 2006.

32. Wang QM, Kang L and Wang XH: Improved cellular immune response elicited by a ubiquitin-fused ESAT-6 DNA vaccine against Mycobacterium tuberculosis. Microbiol Immunol 53 384-390, 2009

33. Pal A and Donato NJ: Ubiquitin-specific proteases as therapeutic targets for the treatment of breast cancer. Breast Cancer Res 16: 461, 2014

34. Rodriguez F, Zhang J and Whitton JL: DNA immunization: ubiquitination of a viral protein enhances cytotoxic T-lymphocyte induction and antiviral protection but abrogates antibody induction. J Virol 71: 8497-8503, 1997.

35. Cao T, Lazdina U, Desombere I, et al: Hepatitis B virus core antigen binds and activates naive human $\mathrm{B}$ cells in vivo: studies with a human PBL-NOD/SCID mouse model. J Virol 75: 6359-6366, 2001.

36. Chen W, Shi M, Shi F, et al: HBcAG-pulsed dendritic cell vaccine induces Th1 polarization and production of hepatitis $B$ virus-specific cytotoxic T lymphocytes. Hepatol Res 39: $355-365,2009$ 